\title{
Ptyctimous mites (Acari, Oribatida) in the fauna of North Africa, against the background of the Palaearctic fauna
}

\author{
WOJCIECH NIEDBAŁA \\ Department of Animal Taxonomy and Ecology, Faculty of Biology, Adam Mickiewicz University, \\ Umultowska 89, 61-614 Poznań, Poland \\ Corresponding author: wojciech.niedbala@amu.edu.pl
}

(Received on 24 November 2011; Accepted on 24 September 2012)

\begin{abstract}
The ptyctimous mite fauna of each zoogeographical region of the world is highly specific and includes many endemic or native species. In Palaearctic Region it comprises 295 known species. The number of widespread species is very low: 14 semicosmopolitan, 16 Holarctic, and 15 pan-Palaearctic. Out of the relatively narrowly distributed species, over half (114) have also been noted as endemic to the Palaearctic Region. Twenty-seven species are known to originate from other zoogeographical regions and have been introduced to the Palaearctic in prooriental and East-Asiatic parts. The fauna of North Africa has a much lower number of endemic species than the neighbouring subregions, but a high number of Palaearctic species and a relatively high number of widespread species. This fauna is completely different from the Afrotropical fauna on the same continent. The majority of common species are West Palaearctic. The fauna of North Africa is much poorer in species than that of North Mediterranean subregion, but both these faunas share a lot of common species, especially many of West Palaearctic origin. Moreover, in North Africa the regional faunas show poor similarity, e.g. the fauna of the western islands (Canary Island and Madeira) is very different from that of Maghreb (Algeria, Morocco, Tunisia).
\end{abstract}

Keywords: Oribatida, ptyctimous mites, North African fauna

\section{INTRODUCTION}

Ptyctimous mites (Acari, Oribatida) are typical soil invertebrates and occur all over the world wherever there is decaying organic matter. They are a cosmopolitan group, commonly found in all terrestrial ecosystems. However, only recently their great diversity has been discovered, mainly at the species level.

The fauna of ptyctimous mites of each zoogeographical region of the world is highly specific and includes many endemic or native species. A comparative analysis of the ptyctimous mite fauna of the regions reveals a very weak similarity as well poor exchange between regions (NiEDBAŁA 2009). The number of common species is very low. 


\section{MATERIAL AND METHODS}

The considerations presented below are based on the material from the comprehensive work on the Palaearctic ptyctimous fauna (NiEdBAta 2011), in which also the methods of analysis and classification of the material are given. North Africa is defined here as the area extending from the western islands (Canary Islands and Madeira) to northern Maghreb (Algeria, Morocco, Tunisia).

\section{RESULTS AND DISCUSSION}

The Palaearctic is a zoogeographic region rich in ptyctimous mites, represented by 295 species (NiedBAeA 2011), but a few more have been found in the Neotropical Region, 305 species (Niedbata 2004), while 240 species in the Australian Region (NiEDBAta 2012). In the neighbouring Oriental and Afrotropical regions, the numbers of known species are 119 and 148, respectively. The lowest number of species (104) have been found in the Nearctic Region (Fig. 1) (Niedbata 2002, 2009).

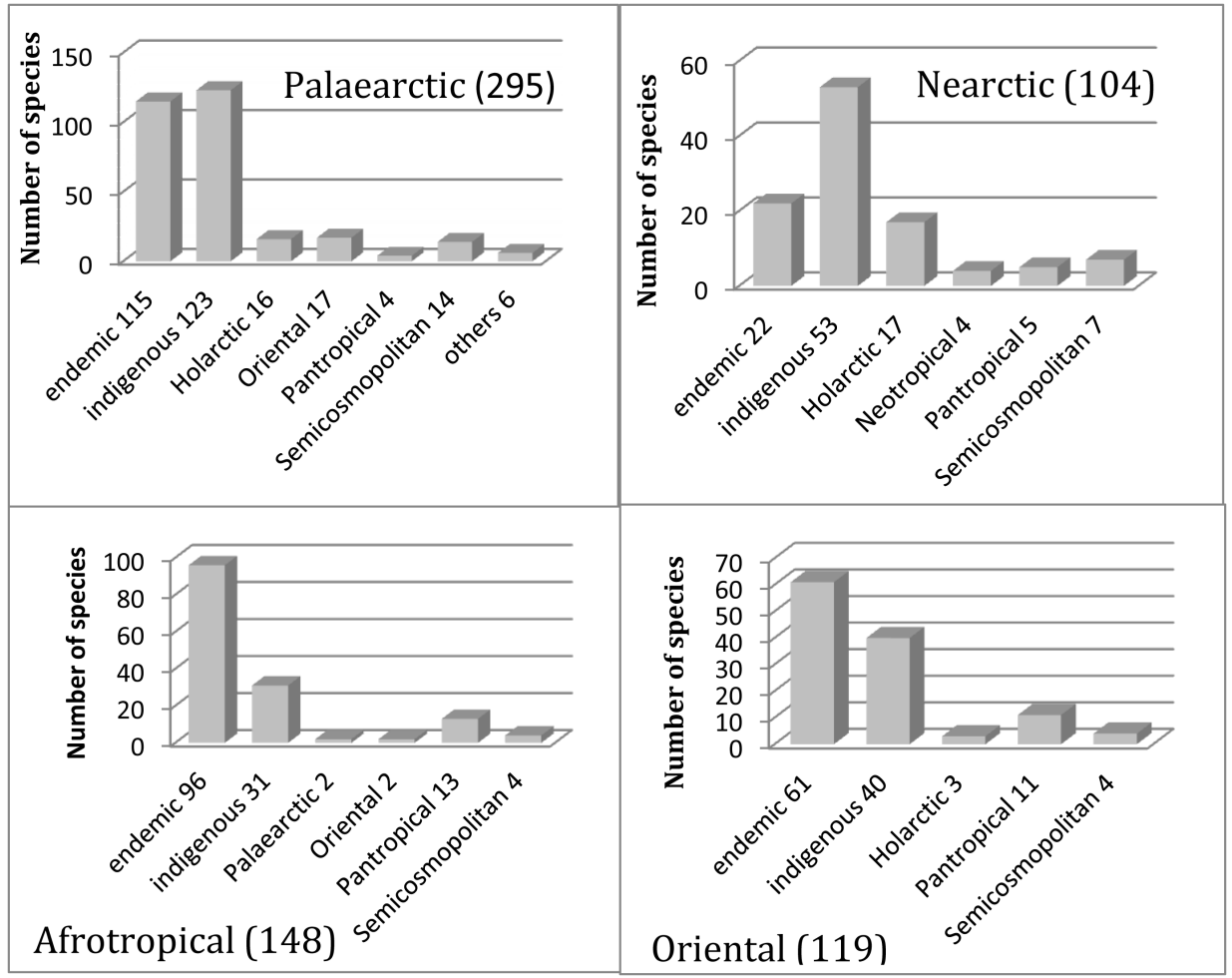

Fig. 1. Contributions of zoogeographical elements to the ptyctimous mite faunas of some zoogeographical regions 
The Palaearctic fauna has a higher proportion (81\%) of restricted (endemic and indigenous) species than the Nearctic fauna (72\%), but the former is similar to the number of species found in the Orient and Afrotropical Region (85\% in each). The proportion of widespread species (semicosmopolitan and pantropical) in the Palaearctic fauna is much lower (6\%) than in the faunas of the Nearctic, Afrotropical and Oriental Regions (11-12\%) (Fig. 1).

In the Palaearctic Region there are 27 species known to originate from other zoogeographical regions and introduced to the Palaearctic. First of all, they are found in prooriental and East-Asiatic parts, and they include four pantropical, four Palaeotropical, 17 Oriental, and one Nearctic species. Moreover, one Neotropical species has been introduced to a palmhouse in the central Palaearctic.

Quite large is the number of widespread species: 14 semicosmopolitan, 16 Holarctic, and 15 pan-Palaearctic. Eight species are somewhat less widespread: three South-Eastern Palaearctic, two Central-Eastern Palaearctic, two South-Western Palaearctic, and one Central-Western Palaearctic (Fig. 2).

Over half of the remaining 215 native species of relatively narrow distribution are endemics (114). In the Western Palaearctic, the highest number of 146 species

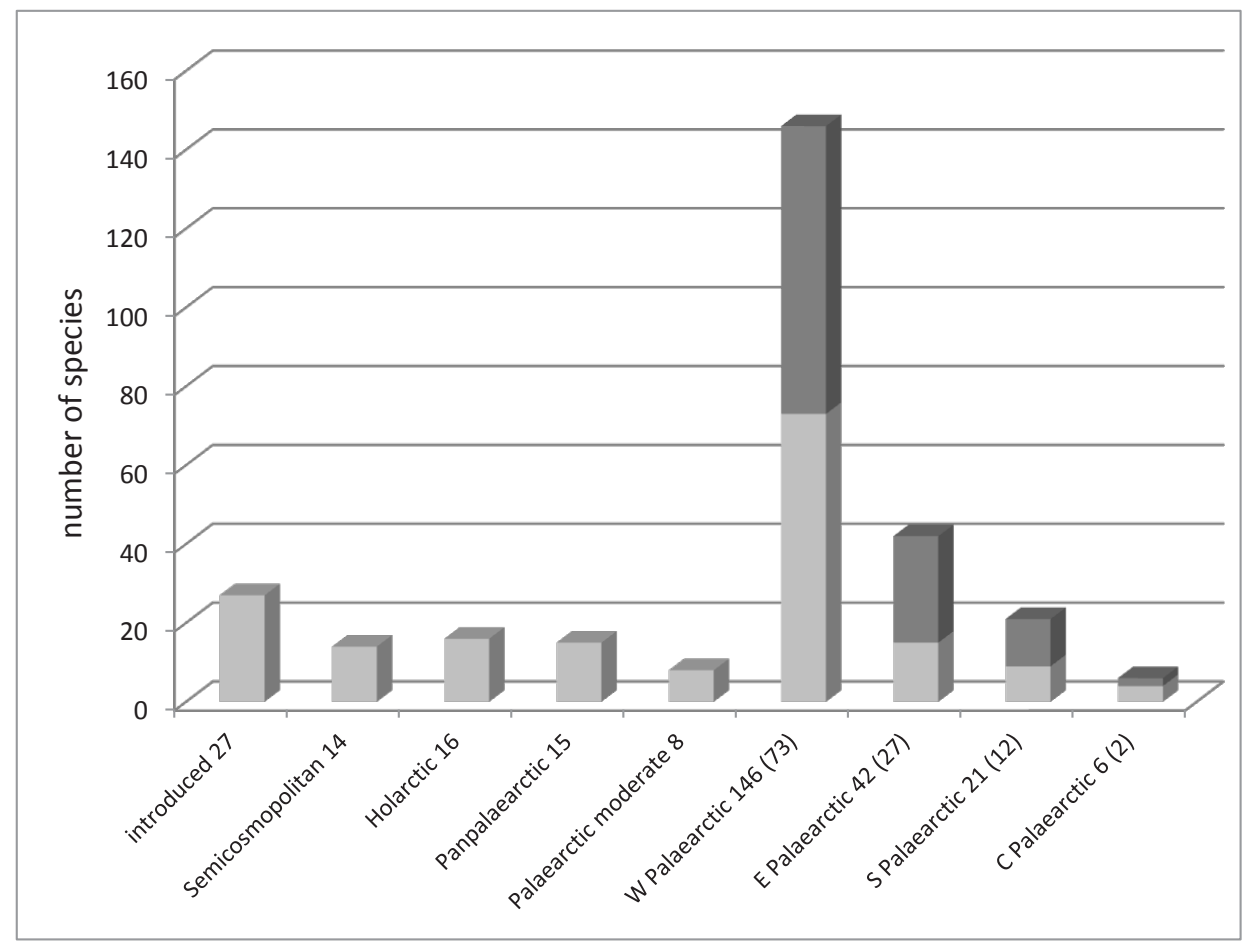

Fig. 2. Contributions of zoogeographical elements to the ptyctimous mite fauna of the Palaearctic (dark colour - endemic). $\mathrm{W}=$ Western; $\mathrm{E}=$ Eastern; $\mathrm{S}=$ Southern; $\mathrm{C}=$ Central 
was found, including 73 endemics. In the Eastern Palaearctic, the number of species was 42, including 27 endemics, while in the Southern Palaearctic, 21 species were recorded, including 12 endemics, and in the Central Palaearctic, only six species, including two endemics (Fig. 2).

Against the background of the knowledge of the Palaearctic fauna it seems interesting to analyse the fauna of North Africa, in the southern Palaearctic. The fauna of North Africa has a much lower number of endemic species (27\%) than the neighbouring regions, but a high number of Palaearctic species (54\%) and a relatively high proportion of species widespread in the Holarctic and semicosmopolitan ones $(18 \%)$ (Fig. 3).

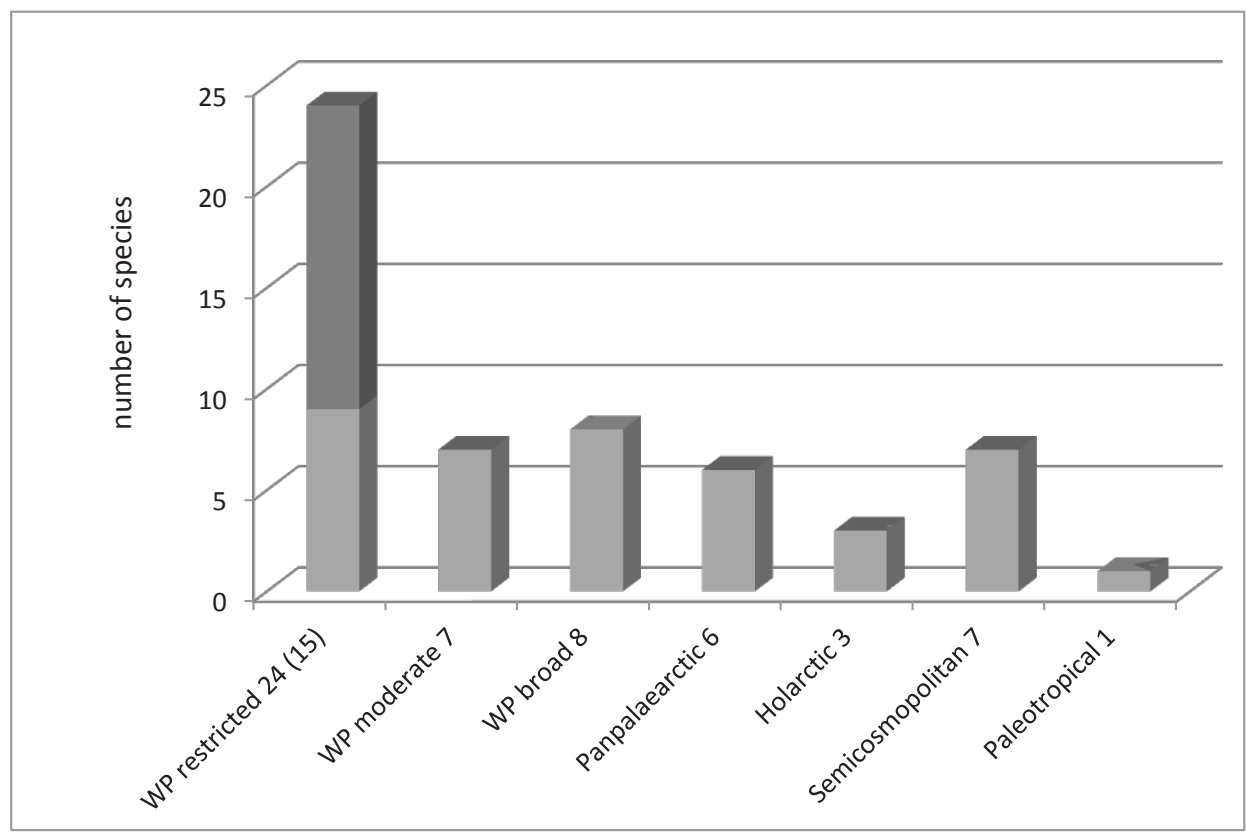

Fig. 3. Contributions of zoogeographical elements to the ptyctimous mite fauna of North Africa (dark colour - endemic) $. \mathrm{WP}=$ Western Palaearctic

Let us compare the North African fauna with that of the North Mediterranean subregion. The fauna of North Africa includes 56 ptyctimous mites, while that of the North Mediterranean subregion comprises almost twice this number, 108 species (Fig. 4). As many as 35 species are common to those subregions, including five semicosmopolitan, two Holarctic, six pan-Palaearctic, and 22 West-Palaearctic (eight widespread pan-European, seven moderately distributed and seven of restricted distribution in the West Palaearctic). 


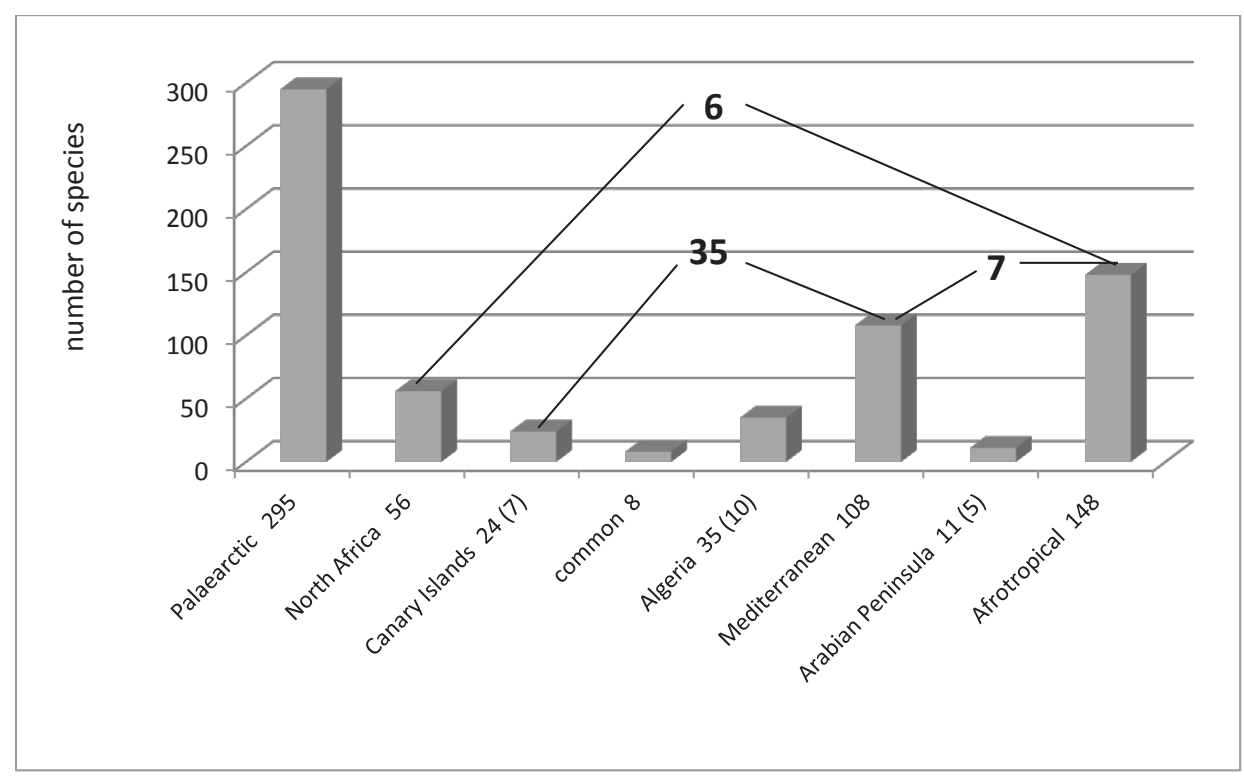

Fig. 4. Numbers of ptyctimous mite species in neighbouring zoogeographical regions and subregions

The fauna of North Africa is completely different from the Afrotropical fauna on the same continent. The Afrotropical fauna has 148 species, of which only six widespread species are shared with North Africa: two of them semicosmopolitan, one Holarctic, two West-Palaearctic broad, and one West-Palaearctic temperate (Fig. 4). The similarity between the faunas of the North Mediterranean (108 species) and Afrotropical (148 species) subregions is also poor. They share only seven common species, including two semicosmopolitan, one Holarctic, two West-Palaearctic broad, and two West-Palaearctic temperate (Fig. 4).

The fauna of North Africa is not uniform. It is very different in the western islands (Canary Islands and Madeira) from that in Maghreb (Algeria, Morocco, Tunisia). The number of species found on the islands is 24 , including 7 exclusive ones: Austrotritia herenessica, Phthiracarus torosus, Steganacarus (Steganacarus) carlosi, S. (S.) guanarteme, S. (S.) similis, S. (S.) tenerifensis, Austrophthiracarus gomerensis. In the continental North Africa, the number of species found has reached 35, among which 10 are exclusive: Acrotritia closteros, Austrophthiracarus costai, Grandjeanoplophora mauritanica, Neoprototritia zachvatkini, Phthiracarus aegypticus, Steganacarus (Steganacarus) grandjeani, Steganacarus (Tropacarus) boulfeka$r i, S$. (T.) callainii), S. (T.) desmeti. Out of the eight common species all are widespread (two semicosmopolitan, one Holarctic, one pan-Palaearctic, and four West Palaearctic, broad and moderate). 
The fauna of the Arabian Peninsula has been poorly recognised, only 11 species have been reported from this area, of which five are endemic, one semicosmopolitan, and five West-Palaearctic (Fig. 4).

\section{REFERENCES}

Niedbala W. 2002. Ptyctimous mites (Acari, Oribatida) of the Nearctic Region. Mongr. Up. Sil. Mus. 4: 1-261.

Niedbata W. 2004. Ptyctimous mites (Acari, Oribatida) of the Neotropical Region. Ann. Zool. 54: $1-288$.

Niedbala W. 2009. Ptyctimous mites of Southern Hemisphere (Acari: Oribatida). Genus 20: $557-562$.

Niedbata W. 2011. Ptyctimous mites (Acari, Oribatida) of the Palaearctic Region. Systematic part. Fauna Mundi 4: 1-472.

Niedbata W. 2012. Ptyctimous mites (Acari: Oribatida) of the Australasian Region with descriptions of thirteen new species. Acarologia, 52: 183-228. 